\title{
Avoiding PBP in Network Mobility Handoff Management
}

\author{
Mageswaran M \\ MS Software Engineering \\ School of Information \\ Technology and Engineering \\ Vellore Institute of Technology \\ Vellore, India.
}

\author{
Manigandan $\mathrm{P}$ \\ MS Software Engineering \\ School of Information \\ Technology and Engineering \\ Vellore Institute of Technology \\ Vellore, India.
}

\author{
Manikandan $\mathrm{K}$ \\ Assistant Professor (Senior) \\ School of Computing Sciences \\ And Engineering \\ Vellore Institute of Technology \\ Vellore, India
}

\begin{abstract}
Mobile Computing has become very important in recent years due to increase in the number of mobile devices. The major issue is providing seamless mobility to them. Mobile IP developed by the IETF allows users to move from one network to another network while having a fixed IP address. Applying Mobile IP in nested architecture results in very low performance with high delay and overhead. In order to overcome this problem IETF Mobile working group has developed Network Mobility protocol (NEMO) for managing mobile Networks. The NEMO protocol is inefficient as it provides high hand-off latencies in nested networks. This is mainly due to the use of Sub-optimal Routing. This type of Routing causes Pinball problem. The solution designed provides low hand-off delays and reduces the pinball problem in mobile networks.
\end{abstract}

\section{General Terms}

Network Mobility, Mobile Computing, NEMO

\section{Keywords}

Nested Architectures, Network Mobility, pinball, hand-off latency

\section{INTRODUCTION}

The usage of wireless devices such as laptops, smart phones etc have been increased during the past few years. The users move from one place to another place and due to this the devices has to support heterogeneous wireless networks. These devices have many limitations in terms of memory, processing speed and battery backup. Eventually these devices can be inter-connected with each other. Within these mobile networks some devices forward the data and support connectivity to other devices. These are termed as Mobile Routers (MR).

Many scenarios of Mobile networks can be formed. They can also be formed in many public places. In some situations mobile network can behave as gateway i.e. they provide Internet access to many other mobile networks. Nested Mobile Networks are also formed. In these situations providing continuous transfer of data to mobile nodes is necessary. Increase in the number of levels and nodes in networks increases the complexity of transfer of data. The existing solutions such as Mobile IP are only used for host mobility. Using Mobile IP in nested architectures leads to poor results with overhead and intolerable delays.

The Internet Engineering Task Force (IETF) proposed a new protocol called NEtwork MObility (NEMO) for mobility management.

\subsection{NEMO Protocol}

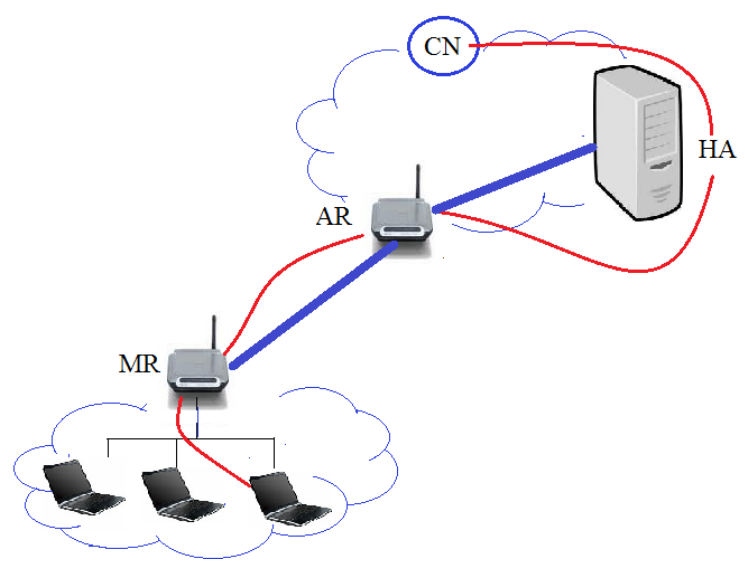

Figure 1: Description of NEMO

A mobile network is a subnet which is mobile and it can be attached to any point in the network. It can only be accessed by gateways called as Mobile Router. A mobile router does not maintain the routes to the point of attachment but maintains a bi-directional tunnel to the Home Agent. A mobile Network can also be nested networks. NEMO [2] is the extension of Mobile IP protocol. In NEMO the Mobile Router (MR) sends Binding Update (BU) to its Home Agent (HA) to update the current location of the mobile network. Whenever the MR enters the foreign network, it is assigned a new temporary address called Care of Address (CoA). This address is obtained in stateless or statefull auto-configuration manner [1]. After the care of address is assigned MR sends a $\mathrm{BU}$ message to register its new location to its corresponding HA. The difference between the Mobile IP [1] and NEMO is that the BU message in NEMO consists of Network Prefix and its Care of Address. By informing about these two data's connection is provided to all nodes in the mobile network through the mobile Router. When any node, say a Correspondent Node wants to communicate with the mobile network node, the corresponding Home Agent intercepts the packet data sent by Correspondent Node and then looks for the entry in its binding cache to the prefix and encapsulation is done and then packets are sent to the CoA of MR. When the packets are sent by the mobile network node to the Correspondent Node the packets are encapsulated by Mobile Router and sent to their Home Agent. Then the home agent forwards it to the Correspondent Node. In the above process all the data packets sent are encapsulated, this is called as Mobile Router-Home Agent Tunneling. Thus the tunneling process leads to delays and overheads. The delay increases as the nesting level is increased. Here the encapsulated packets 
are sent to their corresponding Home Agents of all Mobile Routers of higher level before reaching their destination. This is termed as "Pinball Problem".

\subsection{Hand-off Latency}

Hand-off latency is one of the important parameter in QoS in mobile Networks. Many real time applications and time oriented applications are affected by high hand-off latency. Hand-off occurs when the Mobile Router moves to another Mobile Router across mobile networks. Therefore Handoff delay is defined as the time difference between the last packet received before the handoff and the first one after it. This delay is made of two components: Movement Detection Delay and Registration Delay. Movement Detection Delay is defined as the time required by the node to find out that it has been disconnected from old Mobile Router and to receive the router advertisement message from the new Mobile Router.

Two algorithms [1] are proposed for Movement Detection. The first one depends on the expiration of lifetime period defined as three times the router advertisement interval. This has the average Movement Detection Delay of $2.5 \mathrm{~s}$. Another algorithm is based on the arrival of new router advertisement messages with different prefix and has average delay of $0.5 \mathrm{~s}$. Many solutions are proposed to reduce the Movement Detection Delay. The Fast Hand-off solutions [3] is the most used one. In the Fast Hand-off the mobile node configures a new Care of Address before it switches to a new network.

The second component is the registration delay, it is the time taken for the Binding Update to reach the Home Agent and the data packets are forwarded to the new location of the mobile node. The data packets undergo the pinball problem and they incur heavy losses in nested mobile architecture.

In this paper we minimize the hand-off latency for nested mobile networks by minimizing the registration delay. The next topic discuss about the problems in NEMO.

\section{PROBLEM DESCRIPTION}

Let us consider the figure 2 to discuss about the problems in NEMO. In this figure mobile networks MN1 and MN2 and MN3 are considered. These networks are handled by MR, PMR (Previous Mobile Router) and NMR (New Mobile Router). MN1 is connected to internet through the Access Router AR.MN2 and MN3 access internet through MN1. MN1, MN2 and MN3 constitute a two level nested mobile network.

\subsection{Pinball Problem}

When NEMO is used in Nested Architectures the data packets undergo many MR-HA tunnels. Each mobile Router encapsulates the data packets on the route to the Home Agent. This leads to sub-optimal end-to-end delay and poor performance. When the Movement detection phase is completed the MN undergoes the Registration Phase by sending the Binding Update Message to its Home Agent. The path the data packets travel are

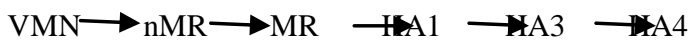

In the above example the nested network with level two is considered. The length of the path increases with increase in the nested level of the mobile networks.

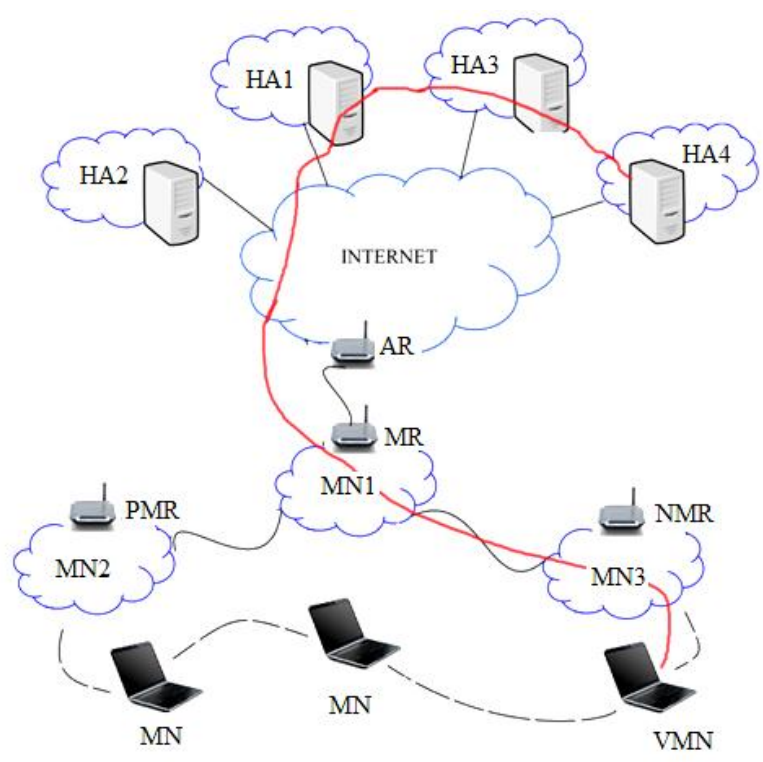

Figure 2: Illustration of Network Mobility

\subsection{Communication within Nested}

\section{Networks}

The tunneling process also affects the communication within the nested networks. As the data packets are encapsulated at each Mobile Router the destination node's address is hidden in the headers. So when the data packets are transferred between the same nested mobile networks, the data packets are unnecessarily traversed the MR-HA tunnel. In the figure when the node MN2 sends data packet to MN3 they go through the following path:

$$
\mathrm{PMR} \rightarrow \mathrm{MR} \rightarrow \mathrm{HA} 1 \rightarrow \mathrm{HA} 2 \rightarrow \mathrm{HA} 3 \rightarrow \mathrm{HA} 1 \rightarrow \mathrm{MR} \rightarrow \mathrm{NMR}
$$

But the nodes are in the same nested mobile network. Thus Route Optimization problem arises due to the pinball problem. In fast handover mechanism Mobile Node asks the router for the new Care of Address before the hand off process. The PMR and NMR exchange signaling messages i.e. Hand-off Initiation and Hand-off Acknowledgement to check whether the new Care of Address in valid or not. With the lack of route optimization problem described above these Hand-off messages undergoes high end to end delay. Due to this there can be loss of packets and connection drops. Even though if the tunnel is established successfully before the connection drop, they are subjected to the pinball problem and cause more delay in the data transfer.

\section{RELATED WORKS}

Jae-Kwon Seo, Sung-Hyun Nam, and Kyung-Geun Lee proposes a fast route optimization protocol [5] to support realtime applications even if the levels of nesting of a mobile network are dynamically changed. If a group with partial levels of nesting in a nested mobile network moves to another mobile network, the TLMR (Top Level Mobile Router) is changed, the route optimization is not achieved or the communication is disrupted. Thus fast routing and route optimization is needed after the handoff in which the TLMR is changed. In the registration procedure when the VMN (Visiting Mobile Node) enters into the mobile network, the addresses of nodes within the MAP (Mobility Anchor Point) 
domain are assigned hierarchically using a subnet Identifier. If the VMN enters into a mobile network, the VMN generates an initial CoA (ICoA) based on MR prefix. The VMN then performs binding-updates to the VMN's HA. The VMN sends a binding-update message including the VMN's CoA and the $\mathrm{HA}$ sends a binding-acknowledgment message to the VMN. If the registration process is completed, the VMN is able to receive the packets through the HA without the registration to the MAP. The $\mathrm{CN}$ sends the packets whose destination address is the VMN's HoA in order to send a packet to the VMN.

The VMN's HA intercepts the packets and checks its own binding-cache entry and tunnel them to the VMN's ICoA. In the proposed scheme, the route optimization is achieved by a process in which the packet passes through the HA of the VMN. The CN does not participate in the route optimization process of the proposed scheme to prevent the binding-update storm problem after the handoff. In existing schemes, if any $\mathrm{CN}$ that does not communicate with the VMN starts a communication, the $\mathrm{CN}$ should repeat the RO procedure. However, a $\mathrm{CN}$ does not participate in the route optimization procedure in the proposed scheme. Therefore, even though a $\mathrm{CN}$ starts to communicate with a VMN of a mobile network, the $\mathrm{CN}$ does not require an extra process.

$\mathrm{Lu}$ Li-Hua, Liu Yuan-an in their paper proposed a idea which solves the pinball problem by an extended prefix information option in RA message. Modifying movement detection, the solution makes a difference between handover inside nested NEMO and outside nested NEMO. By using root MR's CoA as its own CoA, tunnel length is shortened and encapsulated level is reduced. Registration in nested NEMO makes handover latency as short as possible. Router Advertisements contain prefixes that are used for on-link determination and/or address configuration, a suggested hop limit value, etc. Multiple prefixes information option (PIO) [6] can be included in RA message. To solve suboptimal routing, the PIO has been modified by adding a U-bit to explain for which host the prefix is serving. If the $\mathrm{U}$ bit equals to 1 means that mobile host should use this prefix to configure their address and perform its movement detection. Otherwise local fixed host should use this prefix.

M.Sabeur, Jouaber, D.Zeghlache describes a new method for solving the pinball problem called as Light-NEMO+ [7]. The Light-NEMO reduces the number of IP-in-IP encapsulations, resulting in one unique tunnel between the VMN (Visitor Mobile Node)'s Home Agent and the MR that provides access to the entire nested mobile network. The philosophy with Light-NEMO solution is to give the HA the required information to distinguish between the CoA of the MNN and the location where to forward packets. This is achieved by introducing the following functionalities in the MNs, MRs and HAs:

- A new bit, R, is added to RA (Router Advertisement) messages of MRs. It is set to "1" by a MR if it is away from its home network or if it receives a RA message with R set to "1".

- When a VMN or a MR receives a RA with $\mathrm{R}$ set "1", it does not encapsulate packets (generated or received on its ingress interfaces)

- A new CoA option is added to the BU (Binding Update) messages and used to store the CoAs of VMNs.

- When receiving a BU with the CoA option, a MR inserts a new entry in its route table for the BU home address option value. The source address of the BU will be the next hop. This entry will be used to route packets sent to/by the VMN. Then, the MR swaps the source address to its CoA

- The only tunnel in this architecture is established between the TLMR and the HA of the VMN

M Dattani, N Thanthry, T Best, R Bhagavathula and R Pendse propose a route optimization based solution [8] to address the pinball routing problem and reduce the tunneling overhead over the cloud. The solution uses the concepts of port redirection to route traffic to a node within the mobile network. The proposed solution uses the concepts of port redirection to route traffic to a node within the mobile network. The proposed solution eliminates the need for tunneling between the nested mobile devices and their respective home network.

To eliminate the pinball routing problem the proposal suggests building a tunnel between the HA of the mobile network and the top level MR. Forwarding of datagram's between the TLMR and the respective mobile networks is provisioned by port address translation. The proposed solution makes use of three additional bits, one in the agent advertisement; one is the binding update and one in the datagram's. These additional bits are taken from the optional bits in the respective headers. The additional bit used in the agent advertisement, termed as " $\mathrm{N}$ " bit is used to notify all the MRs within the mobile network about the presence of nested mobility. The additional bit used in the binding update, as " $U$ " bit intimates the HA about the address of TLMR and also forces HA to update its binding cache with the new information. In addition, when the "U" bit is set in the binding update packet, the intermediate MRs within the mobile network assign a port address to the source MR and update their respective database. The "O" bit in the datagram's IP header is used to indicate the intermediate MRs including the TLMR that the datagram belongs to a nested mobile network and port address translation needs to be performed.

In [9], authors extend partially HMIPv6 (Hierarchical MIPv6) to support nested mobile networks. The main idea is that the TLMR acts as a Mobile Anchor Point (MAP). In addition to its LCoA (Local CoA) configured as described in [2], each MR configures a RCoA (Regional CoA) based on the TLMR prefix. Outbound packets will be encapsulated using the RCoA to avoid encapsulation by the others MRs. For inbound packets, reference [5] proposes a new header, denoted RH2, to indicate the next hop for each MR in the egress path. In addition to the overhead introduced by the $\mathrm{RH} 2$ header, this solution presents limitations in solving the communications problems within the nested mobile networks.

The above works describes the problems and solutions proposed for the pinball problem. The following section describes the architecture of the proposed system. 


\section{PROPOSED SYSTEM}

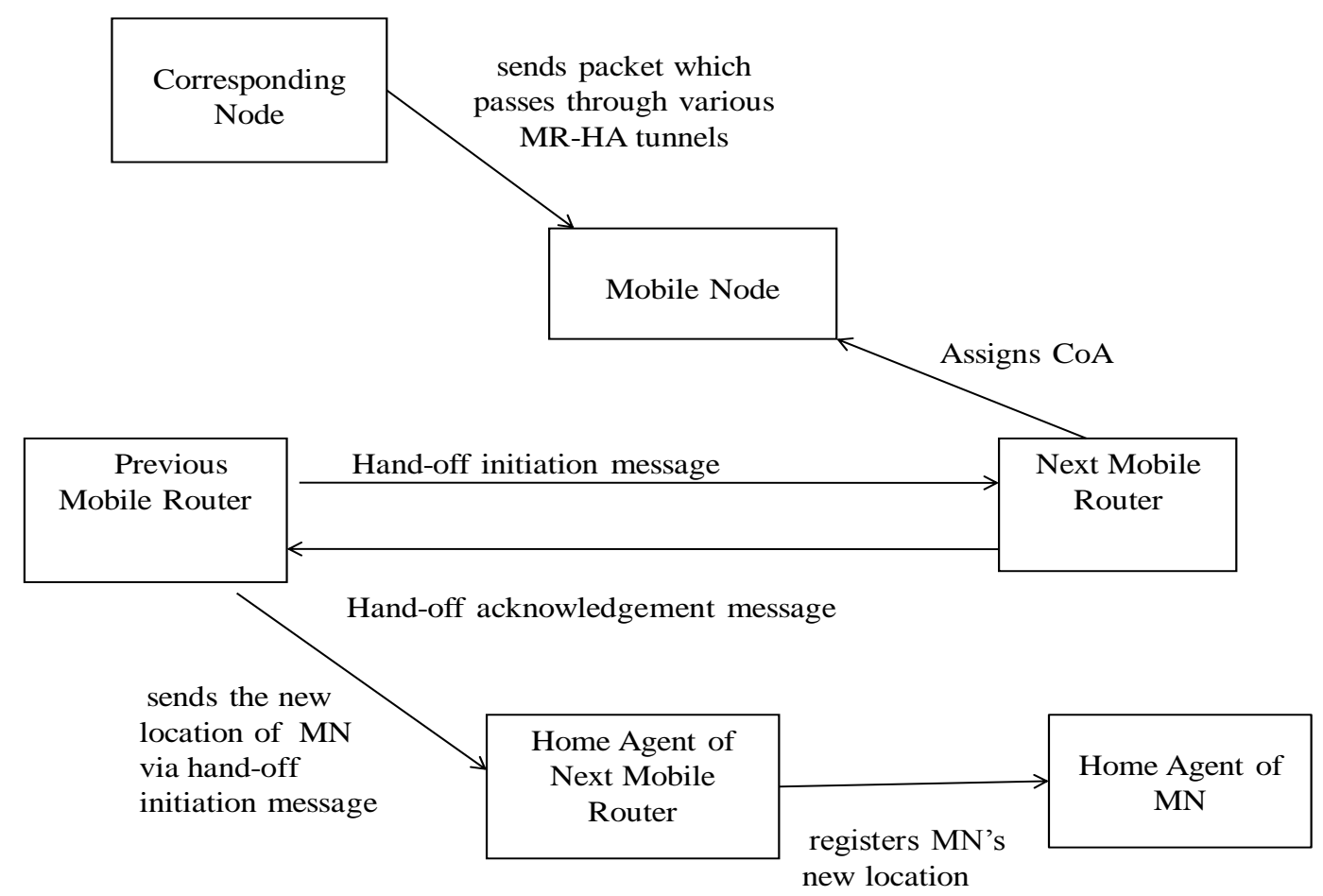

Figure 3: Framework of Proposed Architecture.

In the proposed system, a mobile node is under the coverage area of the previous mobile router. The $\mathrm{CN}$ sends the data to the mobile node; the data passes through the various MR-HA tunnels [11] in the network and reaches the mobile node. After some time the mobile node performs handoff between the routers (PMR and NMR). Previous mobile router initiates the process by sending a hand-off initiation message. This message also passes the various MR-HA messages. This MRHA tunnel is directly proportional to the number of levels in the nested networks. This leads to pinball problem. To solve it the previous mobile router, while sending the hand-off message informs the home agent of next mobile router on the MR-HA path to update the new location of the mobile node to the mobile node's home agent. This enables the mobile node to receive the data without sending binding update to its home agent, thus reducing the hand-off period.

We know that the handoff is made of two components, Movement Detection Delay and Registration delay. The Movement Detection Delay is implemented by the Fast handoff concept [11]. The Registration i.e. the Binding update is sent along with the handoff initiation message, when the handoff initiation message is sent from the previous mobile router it also appends the binding update message to it. This message when reaches the home agent of the next mobile router (due to sub optimal routing), this home agent sends the binding update message to the home agent of the mobile node, thus when handoff occur, the binding update is also done. This is done by embedding the handoff and binding update into a single message.

\section{IMPLEMENTATION DETAILS}

In this section the comparative performance results with NEMO basic support protocol are given. The Simulations are done using the Network Simulator NS2 [4] with mobiwan extension. The performance of hand-off is evaluated in two nested level mobile networks.

The mobiwan supports the implementation of IPv6 and for the simulation purposes we have to create a specialized agent which is combination of Base station and $\mathrm{MN}$ agent. While receiving the Binding Update message the route entries are updated. For this purpose an interface is created between NEMO agent and routing table.

\section{PERFORMANCE}

The simulations are done in the two nested level network. There is a considerable difference in the handoff latency and packet drop in the networks. In case of handoff latency the proposed system .provides the better results than the existing system NEMO. This is because the MN's new location has been updated along with the hand-off initiation message. This enables the $\mathrm{MN}$ to receive data immediately when it comes under a new mobile router. In case of packet loss the proposed system is better than the existing system NEMO. This is because the packet sent experiences only a few MR-HA tunnels in the proposed system. The graphs below give us the comparison of the handoff latency. 


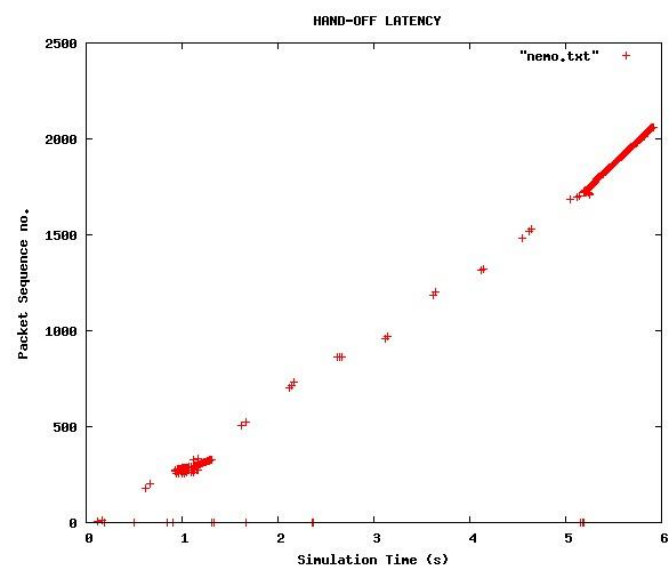

Figure 4: Handoff Performances in NEMO

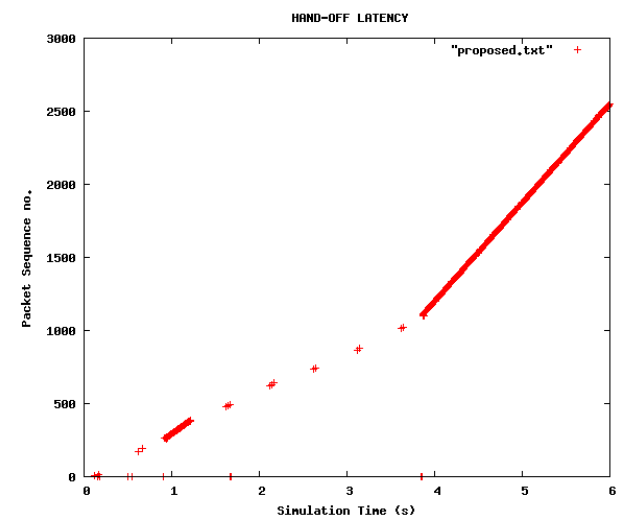

Figure 5: Handoff Performance in proposed System.

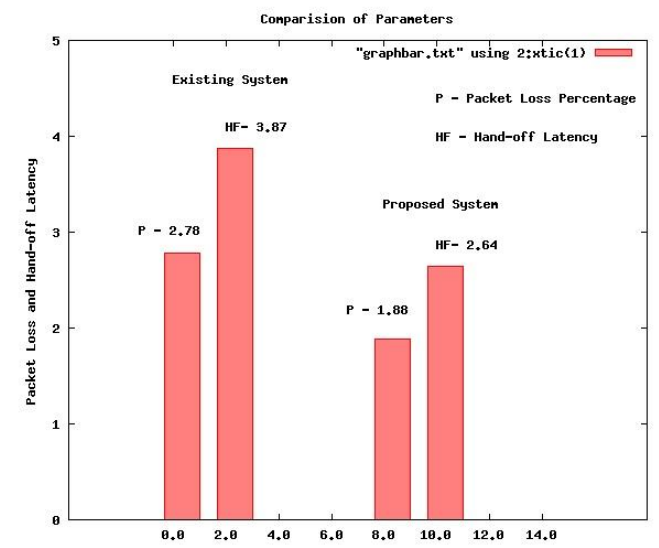

Figure 6: Comparative result.

The bar chart below displays comparative results of Packet loss and Hand-off Latency in both systems.

\section{CONCLUSION AND FUTURE ENHANCEMENTS}

One of the major concerns in Network Mobility is providing seamless data transfer for mobile users. This seamless connectivity is very much affected by Pinball problem and
Hand-off delays that occur in the mobile networks. The solution proposed is effective in reducing the hand-off delays in the wireless scenario. The packet loss in the system also shows a considerable difference when compared to NEMO. The proposed system can be enhanced by adding a security support for preventing the attacks that happens in the networks. For instance the attacker can redirect the mobile user's traffic to another node by changing the Care of Address field [16]. Many such attacks are possible in the networks. These types of problems can be resolved to provide secure data transfer in the mobile networks. Roaming Authentication and access control mechanisms can also be added [12].

\section{REFERENCES}

[1] D. Jhonson "IP Mobility Support for IPv6", RFC 3775. June 2004

[2] Vijay Devarapalli et al. "Network Mobility (NEMO) Basic Support Protocol”, RFC 3963 January 2005.

[3] Koodli, R.(eds.). "Fast handovers for Mobile IPv6". RFC 4068. July 2005.

[4] Network Simulator 2 (NS2), http://www.isi.edu/nsnam/ns/

[5] Jae-Kwon Seo, Sung-Hyun Nam, and Kyung-Geun Lee, "Fast Route Optimization for Dynamic Nested NEMO", International Conference on Parallel Processing Workshops (ICPPW 2007).

[6] Lu Li-Hua, Liu Yuan-an, "Route optimization solution based on extended prefix information option for nested mobility network", IEEE 2007, PP 1792-1796.

[7]. M. Sabeur, B. Jouaber, D.Zeghlache, "LIGHT-NEMO+: Route optimization for LIGHT-NEMO solution", IEEE 2006.

[8]. M Dattani, N Thanthry, T Best, R Bhagavathula and R Pendse, "Route Optimized Nested Mobiltiy Solution Using PAT", IEEE 2004, pp 3105-3109.

[9]. Dongkeun Lee et al. "hierarchical Route Optimization for Nested Mobile Network” AINA'04.

[10]. Hosik Cho, Taekyoung Kwon, Yanghee choi, "Route Optimization using Tree Information Option for Nested Mobile Networks, IEEE 2006, pp 1717-1724.

[11].Min-soo Woo, Youn-Hee Han, Hyo-Beom Lee, Sung-Gi Min, "A Tunnel Compress Scheme for PMIPv6-based Nested NEMO.

[12].Hyung-Jin Lim, Moonseong Kim, Jong-Hyok Lee, DaeHee Seo, Tai M. Chung, "Reducing Communication Overhead for Nested NEMO Networks: Roaming Authentication and Access Control Structure, IEEE 2011,pp 3408-3423.

[13]. Ahmed A.Mosa, Aisha Hassan, Rashid A. Saeed, Othman O. Khalifa, "Evaluation of NEMO-Based Approaches for Route Optimization, ICOM 2011.

[14]. Hu Wu, Jian-de Lu, "Research on Routing Optimization in Nested NEMO", IEEE 2011.

[15]. Azzedine Boukerche, Zhenxia Zhang and Xin Fei, "Reducing Handoff Latency for NEMO-based Vehicular Ad Hoc Networks", IEEE Globecom 2011.

[16]. J. Arkko et all. "Using IPsec to Protect Mobile IPv6 Signaling Between Mobile Nodes and Home Agents" RFC3776. June 2004 